\title{
Big Pharma holt sich blaues Auge
}

Covid beschert den großen Pharmaunternehmen stagnierende Umsätze und Erträge. Österreichs Pharmaunternehmen spielen im Konzert der Großen eine kleine, aber vernehmbare Rolle.

Von Peter Martens

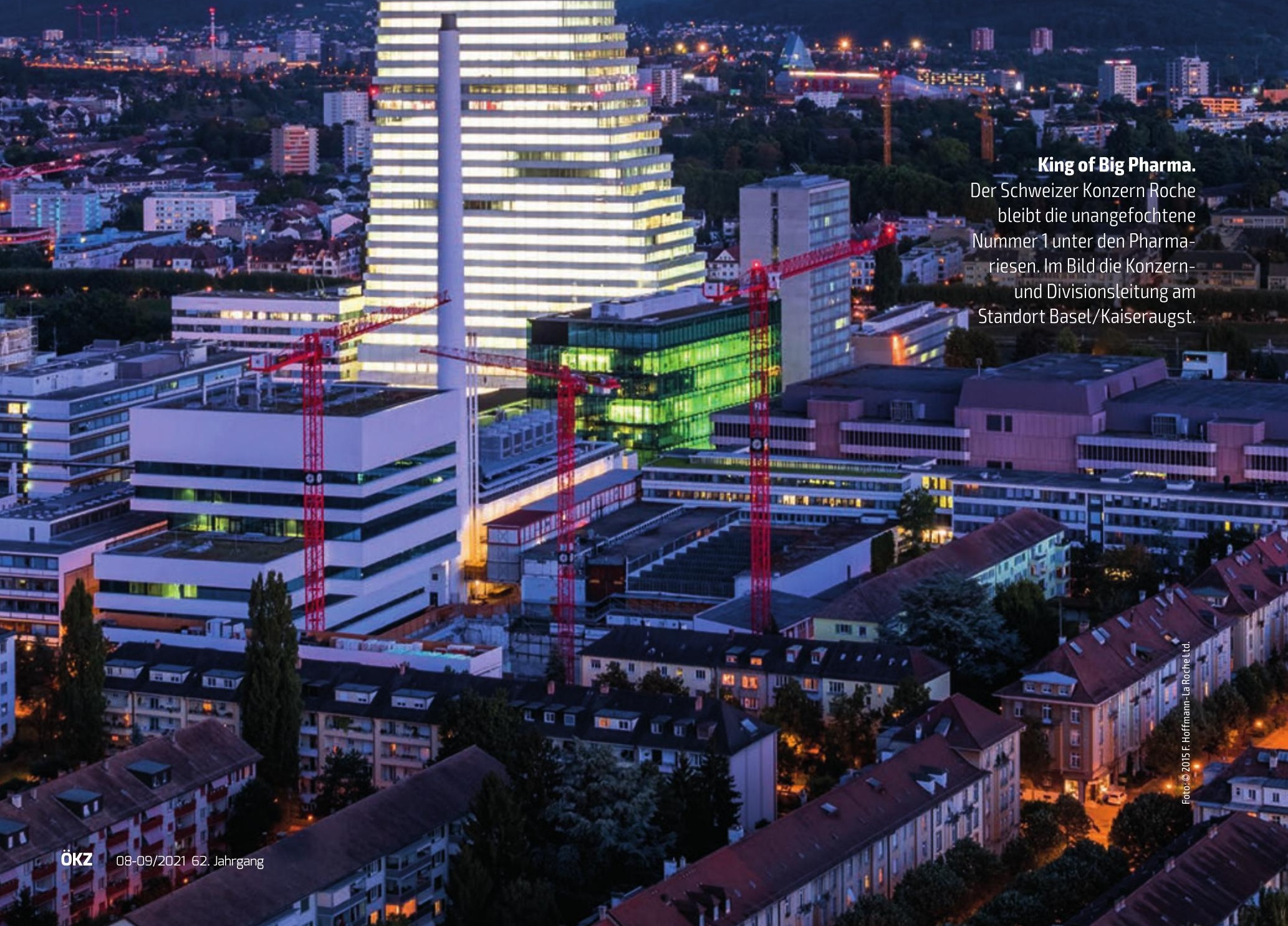


Die Seuche kennt keine Profiteure. Nicht einmal die Pharmabranche konnte im Jahr 1 nach Ausbruch der Pandemie zulegen - zumindest nicht in dem Ausmaß, wie es zu erwarten gewesen wäre. „Pharma ist trotz Umsatzwachstum kein Krisengewinner: Corona hat bei verschiebbaren Behandlungen zu Verzögerungen geführt und laufende Forschungsprojekte beeinträchtigt", meint dazu Erich Lehner, Leiter des Bereichs Life Sciences bei EY Österreich. Dass es 2020 dennoch zu einem leichten Wachstum gekommen ist, erklärt Lehner mit den Erfolgen bei der Entwicklung neuer Medikamente, gepaart mit langfristigen Trends wie der wachsenden und alternden Weltbevölkerung.

Laut einer Analyse von EY, die regelmäßig die Bilanzen der international größten Branchenakteure untersuchen, konnten die 21 weltweit größten Pharmahersteller ihren Umsatz im Krisenjahr um 4,4 Prozent steigern, das Ebit (Betriebsgewinn) verbesserte sich um 2,7 Prozent. Im Vorjahr hatte das gemeinsame EBIT-Wachstum noch um fast 19 Prozent angezogen. Die Coronakrise sei dabei keineswegs ein Wachstumstreiber gewesen, heißt es dazu bei EY Österreich. Im Gegenteil habe die Pandemie zu höheren Ausgaben für Forschung und Entwicklung geführt, was sich wiederum in der hohen Zahl an neuen Impfstoffen und Therapeutika widerspiegele. Auch der deutliche Rückgang der Ambulanztätigkeit in den Spitälern während der Lockdowns hat seine Spuren hinterlassen.

\section{Wachsende Dynamik in Österreich}

Im europäischen Vergleich ist Österreich ein kleiner, aber sehr dynamischer Pharmastandort. „Den Herstellern in Österreich ist es gelungen, langfristig nicht nur einen merklichen Wachstumsvorsprung zum Durchschnitt der Industrie, sondern auch im internationalen Vergleich zu erreichen", heißt es in einer Analyse der Bank Austria. Die heimische Pharmaindustrie in Österreich ist demnach seit 2001 jedes Jahr im Schnitt um 5,8 Prozent gewachsen. Zum Vergleich: Europaweit hat die Pharmabranche in diesem Zeitraum nur um 3,7 Prozent zugelegt und die gesamte Industrie in Österreich lediglich um 2,6 Prozent. Auch im Außenhandel gehört Österreich laut Branchenverband Pharmig netto zu den Exportländern, wobei 46 Prozent des Exports in die EU gehen. Die wichtigsten Akteure sind dabei Ableger oder Akquisitionen der großen Player: Allein die österreichischen Konzerntöchter von Novartis (Sandoz), Boehringer Ingelheim, Takeda (vormals

\section{DIE TOP 10 PHARMAUNTERNEHMEN WELTWEIT NACH NETTOUMSATZ 2020 IN MRD. EURO}

\begin{tabular}{l|c|} 
Roche & $\mathbf{4 3 , 4}$ \\
AbbVie & $\mathbf{4 0 , 2}$ \\
\hline Johnson \& Johnson & 40,0 \\
Merck \& Co. Inc. & 37,7 \\
\hline Bristol-Myers & 37,3 \\
Pfizer & 36,8 \\
\hline Novartis & 34,2 \\
\hline Sanofi & 31,6 \\
GlaxoSmithKline & 27,0 \\
\hline Takeda Pharmaceutical & 26,3 \\
\hline
\end{tabular}

Shire/Baxalta) und Fresenius Kabi beschäftigen aktuell fast zwei Drittel der rund 16.000 Arbeitnehmer der Branche.

\section{Investitionen in F\&E international deutlich gestiegen}

Bei den Branchentrends in Österreich lassen sich dieselben Entwicklungen erkennen, die auch Big Pharma derzeit prägen: Zum einen sind im Coronajahr im Vergleich zum Jahr zuvor die Ausgaben für Forschung um knappe zehn Prozent gestiegen. Die größten 21 Hersteller haben in neue Entwicklungen 103,4 Mrd. Euro investiert, was knapp einem Fünftel ihres Gesamtumsatzes entspricht. „In einer Ausnahmesituation haben sich Pharmafirmen sehr angestrengt, schnell Impfstoffe und Medikamente gegen Corona auf den Markt zu bringen. Viele sind auch wirtschaftliche Risiken eingegangen“, erklärt dazu EY-Experte Erich Lehner.

\section{Forschungs-Fokus auf Onkologie und Immunologie}

Doch der vordergründig starke Fokus auf Covid täuscht. Bei der Analyse der Bilanzen von Big Pharma zeigt sich, dass das Wachstum vor allem von Wirkstoffen gegen Krebs und Immunsystemerkrankungen getrieben ist. So haben die größten 21 in den Bereichen Onkologie und Immunologie um knappe 15 Umsatzprozent zugelegt - laut EY ein neuer Spitzenwert. Allein gegen Krebs befanden sich zuletzt 751 Wirkstoffe in der klinischen Entwicklung oder im Zulassungsverfahren. Das entspricht knapp der Hälfte aller Entwicklungsprojekte großer Pharmakonzerne. Auf dem zweiten Platz folgt das Indikationsgebiet der kardiovaskulären und metabolischen Erkrankungen mit knapp der Hälfe des Umsatzes. An dritter Stelle steht der Bereich der Infektionskrankheiten. Von einer ähnlichen Gewichtung der klinischen Studien in Europa berichtet das österreichische Forum der forschenden pharmazeutischen Industrie (FOPI): Mit 25 Prozent betrifft der größte Teil der laufenden Studien die Onkologie, mit 18 Prozent den Bereich Infektionen und zehn Prozent die Neurologie.

Die Gewinne sind zumindest bei den Großen der Branche stattlich. So erwirtschafteten die 21 größten von ihnen beim Vorsteuergewinn im Schnitt eine Marge von 26 Prozent. Als Margenkönige stechen die Hersteller Gilead und Novo Nordisk mit Werten von über 40 Prozent heraus. Allerdings seien Forschung und Entwicklung mit immensen Kosten verbunden, betonte FOPIPräsident Bernhard Ecker kürzlich bei einer Pressekonferenz. Es würden aber auch die Entwicklungsausgaben immer weiter stei-

\section{PRODUKT-PIPELINES DER TOP 21 NACH THERAPIEGEBIETEN n: 1538 Projekte}

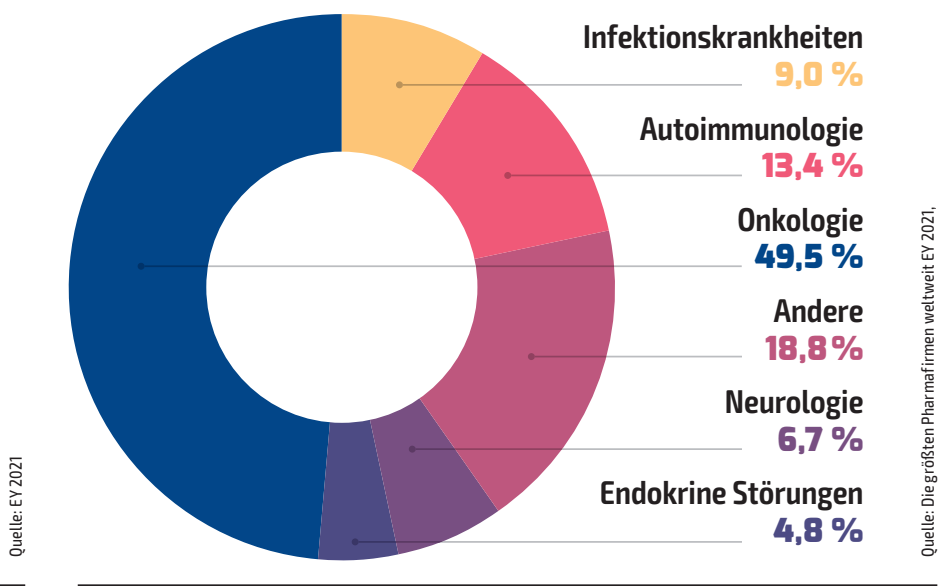


gen und könnten inzwischen bis zu 2,2 Mrd. Euro pro Medikament betragen, so Ecker: „Nur ein bis zwei von 10.000 Substanzen schaffen es bis zur Marktreife."

\section{"Ein Jahr der Innovationen“}

Umso bemerkenswerter, dass in Europa und damit auch in Österreich im Vorjahr 39 Medikamente neu zugelassen wurden. Darunter waren elf Onkologika und vier Medikamente gegen seltene Erkrankungen, darunter Zolgensma als erste Gentherapie gegen Spinale Muskelatrophie bei Kindern, erklärte Christa Wirthumer-Hoche, Leiterin des Geschäftsfelds Medizinmarktaufsicht der Agentur für Gesundheit und Ernährungssicherheit (AGES). Aber auch Mittel gegen Multiple Sklerose, Influenza, HIV, Rheumatoide Arthritis und das Cushing Syndrom kamen im Vorjahr neu auf den Markt. „2020 war ein Jahr der Innovationen“, so Wirthumer-Hoche bei einer Pressekonferenz. „Noch nie wurden so rasch Impfungen oder Medikamente entwickelt. Noch nie gab es so viele Kooperationen zwischen Unternehmen und Forschungszentren und noch nie war die Zusammenarbeit zwischen Forschung, Industrie, Zulassungsbehörden und letztlich auch Regierungen so eng und - meistens - konstruktiv.“

\section{Aus Österreich gegen das Coronavirus}

Österreichische Standorte waren maßgeblich an neuen Entwicklungen beteiligt, etwa Novartis Austria, mit 2,4 Mrd. Euro Umsatz, rund 5.000 Mitarbeitern und knapp neun Prozent Marktanteil in 2020 der größte Pharmakonzern hierzulande. Gerade baut Novartis über seine Generika-Tochter Sandoz den Standort Kundl zum „Zentrum der einzigen durchgängigen oralen Anti-

\section{Berufsbegleitende Universitätslehrgänge}

\author{
Health Care Management mit der \\ Vertiefung Gesundheitsmanagement und \\ Public Health oder Krankenhausmanagement \\ Beginn: 22. November 2021 \\ Abschluss: Master of Science (MSc) bzw. \\ Master of Business Administration (MBA) \\ Lehrgangsbeitrag: EUR 11.900,- (MSc) bzw. EUR 14.900,- (MBA) \\ Kontakt: heike.hoflehner@donau-uni.ac.at, \\ Tel. +43(0)2732 893-2748 \\ www.donau-uni.ac.at/gesundheitsmanagement \\ www.donau-uni.ac.at/krankenhausmanagement
}

\author{
Health Care Management mit der \\ Vertiefung Midwifery \\ Beginn: 22. November 2021 \\ Abschluss: Master of Science (MSc) bzw. \\ Master of Business Administration (MBA) \\ Lehrgangsbeitrag: EUR 11.900,- (MSc) bzw. EUR 14.900,- (MBA) \\ Kontakt: claudia.lehensteiner@donau-uni.ac.at, \\ Tel. +43 (0)2732 893-2743 \\ www.donau-uni.ac.at/midwifery \\ Donau-Universität Krems \\ Die Universität für Weiterbildung

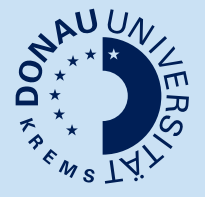

biotika-Produktionskette in Europa“ aus. Der Konzern investiert dafür 100 Mio. Euro, 50 weitere Mio. Euro bekommt der Schweizer Pharmariese als Beihilfen aus dem heimischen Staatsbudget. Heuer im Frühjahr gab Novartis den Ausbau des Tiroler Standorts zu einem „Kompetenzzentrum für die Nukleinsäure-Produktion“ sowie den Einstieg in die Impfstoffproduktion gegen Covid-19 bekannt. Geplant ist die Belieferung des deutschen Impfstoffherstellers Curevac von Kundl aus. Auch wenn es dabei zuletzt Rückschläge gab, betonte Novartis-Vorstand Harry Kirsch Ende Juli, auch andere Hersteller wie Biontech und Pfizer beliefern zu können: „Wir sind nicht auf den einen oder anderen beschränkt.“

\section{Weltweit einzige Forschungsprojekte mit inaktivem Corona-Virus}

International für Schlagzeilen sorgt auch der österreichisch-französische Hersteller Valneva, der sich bei der Entwicklung eines Corona-Impfstoffs für einen anderen Weg als die Konkurrenz entschieden hat: Der Impfstoffkandidat VLA2001 enthält inaktivierte Sars-Coronaviren-2 zusammen mit den Impfstoffverstärkern Alum und CpG 1018. Er wird bereits in einer klinischen Phase3-Studie mit rund 4.000 Teilnehmern in Großbritannien getestet, wo seine Wirksamkeit mit dem bereits zugelassenen Impfstoff von AstraZeneca verglichen wird. Mit dieser Methode könnte eine viel längere Immunisierung erreicht werden sowie die Behandlung von besonderen Zielgruppen, wie Kinder, Schwangere und Menschen mit Vorerkrankungen, verbessert werden. Hat Valneva damit Erfolg, könnte im vierten Quartal ein Antrag auf Zulassung folgen. Größere öffentliche Bekanntheit hat in der Coronakrise auch der in Klosterneuburg ansässige Hersteller Polymun erlangt, der inzwischen Biontech und Pfizer beliefert. Die Niederösterreicher produzieren unter anderem Lipid-Nanopartikel für den führenden europäischen mRNA-Impfstoff und haben dafür ihre Kapazitäten allein heuer um ein Viertel erweitert. Bis zum Sommer hat Polymun 20 statt der geplanten 15 Mio. Dosen des Materials an die europäischen Standorte von Biontech und Pfizer ausgeliefert. Bekannntlich stammt auch einer der drei Gründer von Biontech, der Immunologe Christoph Huber, aus Österreich.

\section{Bekannte Schwierigkeiten am Standort Österreich}

Die Coronakrise hat den europäischen Herstellern schmerzhafte Grenzen aufgezeigt. Etwa 60 Prozent der weltweit produzierten aktiven pharmazeutischen Wirkstoffe kommen aus China und Indien. Bei den Antibiotikawirkstoffen produzieren diese beiden Länder rund 80 Prozent. Österreichs Pharmaindustrie produziere dagegen nur 37 Prozent der Vorleistungen im Inland und sei in acht Vorleistungssektoren komplett von Importen abhängig, so eine heuer publizierte Studie des Economica-Instituts. China habe den Wert pharmazeutischer Patente erkannt, so Economica-Chef Christian Helmenstein. Österreichs Anteil an den weltweiten Patentanmeldungen sei dagegen seit 2010 schrittweise gesunken, in den vergangenen zwei Jahren jedoch mit 0,2 Prozent stabil geblieben. Trotzdem seien hier weitere Anstrengungen der Industrie und Förderungen des Staates notwendig. Die Politik reagiert in bewährter Weise: Es gibt bereits eine Arbeitsgruppe. Life Science ist einer der sieben zentralen Pfeiler der „Standortstrategie 2040“, die im Mai vorgestellt wurde. Der Schwerpunkt Life Science bedeute, die vorhandene Expertise zu nutzen, um im Gesundheitsbereich wieder Produktion nach Österreich zu holen, hieß es bei der Präsentations-Pressekonferenz. Die Voraussetzungen wären gegeben. 